\title{
Detecting Differences between Clinical Presentations in ADHD through the Cognitive Profile Obtained from WISC-IV
}

\author{
Ignasi Navarro Soria ${ }^{1, *}$, Javier Fenollar Cortés ${ }^{2}$, Rocio Lavigne Cerván ${ }^{3}$, Rocio Juarez Ruiz de Mier ${ }^{3}$ \\ ${ }^{1}$ Department of Development Psychology and Teaching, Faculty of Education, University of Alicante, Spain \\ ${ }^{2}$ Department of Evolutionary Psychology and Education, Faculty of Psychology, University of Murcia, Spain \\ ${ }^{3}$ Department of Evolutionary Psychology and Education, Faculty of Education, University of Malaga, Spain
}

Copyright $@ 2017$ by authors, all rights reserved. Authors agree that this article remains permanently open access under the terms of the Creative Commons Attribution License 4.0 International License

\begin{abstract}
Objective: The current study explores whether WISC-IV cognitive profiles could allow to differentiate between presentations of ADHD. Method: A clinical sample of 216 subjects aged between 6 and 16 years and distributed into 2 subgroups (ADHD inattentive type group aged $\mathrm{M}=$ 8.5, $\mathrm{SD}=2.4$; and ADHD combined group $\mathrm{M}=10.1, \mathrm{SD}=$ 2.6) was recruited for the study. Results: Processing Speed Index mean score was significantly higher $(\mathrm{F}(1,214)=14.7$, $\mathrm{p}<.001, \mathrm{~d}=0.52)$ in ADHD-Inattentive group $(\mathrm{M}=90.7$; SD $=12.1)$ than ADHD-Combined group $(\mathrm{M}=97.7 ; \mathrm{SD}=14.7)$. Furthermore, PSI was negatively associated with "Inattention" dimension $(\beta=-.21, \mathrm{p}<.001$ family and $\beta=$ $-.19, \mathrm{p}<.001$ teachers) while it was positively associated with "Hyperactivity/Impulsivity" dimension $(\beta=.27$, $\mathrm{p}<.001$ family and $\beta=.33, \mathrm{p}<.001$ teachers). Conclusions: The higher inattentiveness the lower PSI mean score, while a higher hyperactivity/impulsivity mean score would reduce the impact caused for inattentiveness. Thus, ADHD-I profile would tend to show a significant lower PSI mean score than ADHD-C.
\end{abstract}

Keywords TDAH, WISC-IV, Working Memory, Processing Speed, Instrumental Study

\section{Introduction}

Attention-Deficit/Hyperactivity Disorder (ADHD) is the most common neurobiological disorder in children and adolescents, being one of the main causes of consultation in both Mental Health Units and schools [1]. ADHD diagnosis is based primarily on behavioral symptoms, and is characterized by a persistent pattern of inattention and/or hyperactivity/impulsivity symptoms that occurs before the age of 12 years in at least two different contexts, that is not explained by the presence of another mental disorder and that also interferes in social, academic or occupational functioning [2]. The expression of clinical symptoms may cause a negative impact on the psychosocial development and academic skills of the subject affected by the disorder [3].

Depending on the presence of the main symptoms of the disorder, three diagnostic presentations are established: ADHD with predominance of inattentiveness, ADHD with predominance of hyperactive/impulsive behavior, or ADHD Combined-Type. Despite all the efforts aimed at the standardization, there are no precise data on when the behaviors associated with the disorder, frequency, intensity, duration, interference, and degree of impairment caused by ADHD symptoms are sufficient to be considered inappropriate [4].This means that the diagnosis mainly rests on subjective judgments coming from observers that in many cases are not experts, that are interpreted differently in each case and which are subject to cultural biases [5]. In fact, the use of the diagnostic method is at the discretion of the clinician, who may use a battery of diagnostic tests or may simply make a diagnosis based on the description of the symptoms that were detected by different relatives and professionals who observed the patient in both family and school contexts [6].

This disorder leads to serious problems for those affected by it both in the academic environment [7] and in the home or in the workplace [8]. For this reason, it is of great importance to develop the most appropriate strategies and tools to support the diagnosis and to reduce the possibility of errors in the process [9].

Research has shown that ADHD produces characteristic changes in different areas of cognitive development such as working memory [10-14] executive functions [15-17], processing speed [18], general intelligence quotient [19] and psychomotor speed-coordination [20]. Recent studies 
suggest that these differences in cognitive functioning could help in guiding the diagnosis towards discriminating between the two main types of ADHD: Combined-Type and Inattentive-Type [21].

One of the tests aimed at supporting the diagnosis that is traditionally used to corroborate this complex judgment the WISC-IV [21-24], together with its predecessor (the WISC-III) that also played an important role in this task [18].

Several studies have found that children diagnosed with ADHD and evaluated by the WISC-IV show a characteristic pattern in cognitive development [21, 25]. Although in general intellectual functioning they get close to the normative score ranges [26], a moderate effect size in group differences has been noted regarding the indexes of Processing Speed (PS) and Working Memory (WM) whereas, on the other hand, they show an optimal level in tests related to both Verbal Comprehension Index (VCI) and Perceptual Reasoning Index (PRI) [18].

Recent research suggests that there are differences in the cognitive profile of ADHD subtypes [25-26]. Examples in the body of literature have highlighted Arithmetic, Digits, Symbols and Keys Tests [27], as those tests in which some children who are diagnosed with ADHD obtain much lower scores compared to the rest of the scores in WISC-IV Full Scale tests. Further, when there are relatively low scores in these indexes and tests, they define both the cognitive pattern and the subtype of children with ADHD.

The objectives of the present study are, on the one hand, to explore the relationship between the clinical and cognitive profiles, in a broad sample of subjects diagnosed with ADHD. Put differently, the intensity with which the clinical symptoms associated with ADHD are presented and the relationship they maintain with the measured cognitive profile, through the main WISC-IV indexes. On the other hand, the aim is to confirm the hypothesis by taking into account the "cognitive step" that different investigations have related to the ADHD [18, 21, 25]. This latter step consists of significantly lower scores in the Working Memory Index (WMI) and Processing Speed Index (PSI) compared to the scores of the subject evaluated in the Verbal Comprehension Index (VCI) and Perceptual Reasoning Index (PRI), can establish a predictive relationship between the ADHD dimensions (inattention and hyperactivity/impulsivity) and the Working Memory Index (WMI) and Processing Speed Index (PSI) as measured by WISC-IV [28] a predictive relation that could be of great help in the diagnosis of ADHD and its clinical presentation.

\section{Methods}

\subsection{Participants}

The clinical sample was composed of 216 subjects (171 men and 45 women) between the ages of 6 and 16 years, with a mean age of 9 years. All participants are included in the clinical sample from Child and Adolescent Mental Health and Neuropediatric Units, psycho-pedagogical cabinets and associations of families affected by ADHD in the provinces of Valencia, Alicante, Murcia and Malaga in Spain. Previously, they had been diagnosed with ADHD by the clinical team of their corresponding Child and Adolescent Mental Health Unit and, in the case of being treated at the pharmacological level (140 subjects of the total sample), this treatment was based on methylphenidate. The following exclusion criteria were established: not having had a previous ADHD diagnosis established by a specialist in child psychiatry; child neurologist or clinical psychologist outside of this study; not showing clinically significant ADHD symptoms at the time of the study; having an IQ $<80$ (as measured by the WISC-IV); presence of significant symptoms of severe mental disorders (psychosis, major depression, etc.) or other disorders with symptoms that are consistent with ADHD; suffering from severe medical conditions (epilepsy, brain damage, etc.).

\subsection{Instruments}

The technical tools used in this study were:

1. Strengths and Difficulties Questionnaires for Parents and Teachers [29]: SDQ is a 25-item screening measure with and emotional symptoms, conduct problems, hyperactivity, peers problems, and prosocial scales. Each item was rated on a 3-point frequency of occurrence scale for the past 6 -months $(0=$ "not true"; 1 = "somewhat true"; 2 = "certainly true"). Given the five SDQ subscales demonstrate adequate psychometric properties in earlier studies [30]. Cronbach's alphas for our sample for parents varied from .68 (conduct problems) to .75 (emotional symptoms), and was .68 for hyperactivity. The Cronbach's alphas for teachers varied from .67 (emotional symptoms) to .79 (hyperactivity). For the study, we used the "hyperactivity" and "prosocial behavior" subscales within the clinical limits stipulated by the scale, except for those subjects for whom the inattention dimension was predominant, in which case the limit was established in a score of 4 and above [31]. Those subjects who did not obtain a clinically significant score either from their family or their education center were excluded from the clinical group.

2. ADHD-Rating Scale DSM-IV [32]: This is a Likert-type 18-item scale based on DSM-IV diagnostic criteria for ADHD, which measures the dimensions "inattention" and "hyperactivity/impulsivity". Within the age limits, we used the Spanish normative values of this scale together with the values established by the scale itself, deeming a score of $\geq \mathrm{P} 90$ in each dimension as clinically significant. For the dimension "inattention", Cronbach's alphas were .80 and .89 (parents and teachers, respectively) whereas for the dimension "hyperactivity/impulsivity" they were .81 and .88 
(parents and teachers).This tool permitted the creation of clinical subtypes within the total study sample.

3. Short Version of the Spanish Version of the Conner's Rating Scale 3rd Edition for Parents and Teachers [33]: Family and teachers completed the Conners-3 (S), which measures inattention (5 items), hyperactivity/impulsivity (6 items),

defiance/aggression (5 items), peer relationship problems (5 items), and learning problems/executive functions (10 items for family form and 6 items for teachers form). Each item was rated on a 4-point frequency of occurrence scale for the past month $(0=$ not true at all; $1=$ just a little true; 2 = pretty much true; 3 = very much true). Previous studies provide support for the psychometric properties of the Conners-3 [33]. Cronbach's alphas for family varied from .62 (learning problems scale) to .91 (hyperactivity scale), and was .85 for inattention scale, and from 0.86 (defiance/aggression scale) to 0.91 (peers relationship problems scale) for teachers. Cronbach's alphas for teacher were .87 and .89 for inattention and hyperactivity/impulsivity, respectively.

4. Semi-structured family interview: Research team members carried out semi-structured interviews with the families in order to test whether the subject included in the sample met the criteria of the Psychodiagnostic Manual DSM-V [2]. In those cases, in which the clinical team had deemed it appropriate, based on vague or incoherent information on the part of the relatives, an interview with tutors and specialists involved in the intervention with the subject at school was also carried out.

5. Wechsler Intelligence Scale for Children, IV Edition: This is a cognitive test for children aged between 6 and 16 years. For this study, we used the Spanish version to assess general intellectual functioning (FSIQ) through10mandatorysubteststhat generate four indexes: Verbal Comprehension Index (VCI), Perceptual Reasoning Index (PRI),Working Memory Index (WMI) and Processing Speed Index (PSI), and 5 supplemental subtests that can replace any of the mandatory tests or enrich the exploration by providing additional information. The General Ability Index (GAI) and the Cognitive Proficiency Index (CPI) were also calculated. The GAI is a composite score based on some of the verbal and nonverbal subtests that are used to calculate the VCI and the PRI. On the other hand, the CPI summarizes in a single score the results of both WMI and PSI. For this study no additional subtests were included. This scale has demonstrated the adequacy of reliability and validity coefficients in both the American and Spanish versions. Therefore, the reliability of the WISC-IV in its Spanish adaptation, presents coefficients in the range of 0.86 (IVP) to 0.95 (CIT) for the split-half method, which are similar to the coefficients found in the American version, demonstrating the high consistency and stability of the test. Further, in order to determine validity, different procedures were used, including theoretical considerations, internal structure of the test, inter-correlational studies, factorial analysis studies, and examples of evidence based on the WISC-IV relationship with other evaluations. On the other hand, correlation analyses showed significant inter-correlations between the tests corresponding to each of the indices or factors, in agreement with the results found in the original version of the test. In general, the reliability and validity tests performed in the Spanish version of the instrument support the use of the WISC-IV for this context.

According to the preliminary diagnosis that was undertaken by clinical staff of the public health system and the results in the experimental diagnosis after applying the described battery of instruments, two clinical subgroups from the clinical sample were formed: (1) ADHD Combined-Type and (2) ADHD predominantly Inattentive-Type.

\subsection{Procedure}

First, researchers contacted the coordination staff of several Children's Mental Health and Neuropediatric Units from the territories in which the samples were to be selected. Likewise, they also resorted to different local associations of parents with children who are affected by ADHD. Through these two channels, the aims of our study and a request for participants were presented. For a subject to be part of the sample, it was established as a mandatory requirement that they were to have been previously diagnosed with ADHD by a clinical practitioner.

Relevant data collection was carried out by three different administrators, all of them using the same protocol that included the previously described tools. After requesting authorization from the relevant authorities, psychometric assessments were conducted in the subjects' educational centers, as well as in the headquarters of the higher education university centers that were participating in the study and in private psychology clinics that collaborated as disinterested help. Upon further analysis, the obtained results were returned to the families during individual interviews and these were accompanied by a technical report.

The team contrasted the previous clinical diagnosis with the experimental diagnosis for every participant, and only those participants who scored positive results in ADHD diagnosis for both processes were included in the research sample. Legal guardians gave their informed consent for the participants to participate in the experiment. The Bioethics Committee at the University of Murcia, Spain approved the protocol of this study.

\subsection{Analysis Strategy}

The analytical strategy of this paper consists of four phases: 
i. For each one of the WISC-IV indexes, the normal distribution of the data was checked. Using bivariate correlations we explored whether there was a relationship between age and index scores on tests, and using analysis of variance (ANOVA) we checked whether there were significant gender differences and also the size of the effect of such differences.

ii. We also explored the relationship between the primary and secondary indexes of the WISC-IV, and the main symptoms of ADHD as measured both by parents and teachers. Multiple regression analysis was applied to explore the extent to which WISC-IV indexes predict ADHD symptoms. Results were corroborated by taking gender and medication into account.

iii. ANOVAs were conducted to explore differences in ADHD scores depending on which of the disorder's nominal groups the differences belong.

iv. A new variable was calculated from the difference between the scores of Working Memory Index and Processing Speed Index. Correlation and regression analysis, as well as ANOVAs, were applied between this new variable and ADHD symptoms.

\section{Results}

The normal distribution of values was checked for each of the IQ indexes (Kolmogorov-Smirnov test $p>.05$ ). No correlations between age and IQ levels were found, except for Verbal Comprehension Index, although this correlation was low $(r=.18 ; p<.05)$. No significant differences between genders were found, except for Processing Speed Index $(p<.01 ; F=7.12 d=0.45)$ in which the female group obtained a total average score $(M=99.1, S D=12.2)$ which was higher than the total average score for the male group ( $M$ $=$ 93.2, $S D=13.9$ ). Significant differences between medicated and non-medicated subjects were found only in Total IQ, the medicated subjects having obtained a higher score $(M=100.2, \mathrm{SD}=14.0)$ than the non-medicated subjects $(M=95.8 ; S D=11.8)$, but with a low effect size $(F$ $=3.29 ; p<.05 ; d=0.34)$. We have deemed a correlation of 0.1 as a low size effect, a correlation of 0.3 as a medium effect and 0.5 as a large effect. Full socio-demographic data can be seen in Table 1 .

Table 1. Socio-demographic data of the sample by clinical groups and main indices of the test WISC-IV (ANCOVA, age adjusted)

\begin{tabular}{|c|c|c|c|c|}
\hline & TDAH-Combined & TDAH-Inattentive & $F^{a} / \chi$ & $d$ \\
\hline$n$ & 105 & 111 & & \\
\hline Age & $8.5(2.4)$ & $10.1(2.6)$ & $26.94 * * *$ & 0.64 \\
\hline Gender (\%male) & 78.1 & 79.3 & .01 & \\
\hline Medicated (\%) & 63.8 & 65.7 & .53 & \\
\hline \multicolumn{5}{|l|}{ ADHD-RS-Family } \\
\hline Ina. & 8.1(1.1) & $7.6(1.4)$ & 0.63 & \\
\hline Hyp/Imp & $7.6(1.1)$ & $2.2(1.7)$ & $98.25 * * *$ & 3.77 \\
\hline \multicolumn{5}{|c|}{ ADHD-RS-Teachers } \\
\hline Ina. & $7.5(1.5)$ & 7.3(1.4) & $7.01^{* *}$ & 0.14 \\
\hline Hyp/Imp & $6.3(2.7)$ & $2.2(2.5)$ & $596.68 * * *$ & 1.58 \\
\hline VC & $104.5(13.1)$ & $101.4(14.2)$ & 0.63 & \\
\hline PR & $100.4(14.2)$ & $100.9(12.6)$ & 0.49 & \\
\hline WM & 87.9(13.1) & $90.9(14.3)$ & 1.91 & \\
\hline PS & $97.7(14.7)$ & $90.7(12.7)$ & $14.7^{* * *}$ & 0.52 \\
\hline FSIQ & $100.2(13.5)$ & $95.8(12.5)$ & $3.29 *$ & 0.34 \\
\hline GCI & 99.2(13.4) & $102.4(12.9)$ & 0.89 & \\
\hline CCI & $90.9(14.6)$ & $88.4(13.6)$ & 0.96 & \\
\hline
\end{tabular}

Notes. Ina = Inattention; Hyp / Imp = Hyperactivity / Impulsivity; VC = Verbal Comprehension; PR = Perceptual Reasoning; WM = Working Memory; PS = Processing Speed; TIC = Total Intellectual Coefficient; GCI = General Capacity Index; CCI = Cognitive Competence Index.

${ }^{*} p<.05 ; * * p<.01 ; * * *<.001$ 


\subsection{Relationship between WISC-IV Indexes and ADHD Symptomatology}

Significant correlations were found only between Processing Speed and Hyperactivity scores (Table 2) both for parents $(r=.25 ; p<.01)$ and teachers $(r=.28 ; p<.01)$. Understandably, these significant positive correlations also occurred, even if to a lesser extent, in CPI (for both, $r=.18$; $p<.05)$.

Table 2. Correlations between WISC-IV Indexes and ADHD symptomatology

\begin{tabular}{|c|c|c|c|c|}
\hline & \multicolumn{2}{|c|}{ Family } & \multicolumn{2}{c|}{ Teachers } \\
\hline & Ina. & Hyp/Imp & Ina. & Hyp/Imp \\
\hline VC & -.02 & -.09 & -.06 & .05 \\
\hline PR & -.06 & -.07 & -.01 & .02 \\
\hline WM & -.04 & -.05 & -.10 & -.01 \\
\hline PS & -.11 & $.27^{* *}$ & -.12 & $.25^{* *}$ \\
\hline FSIQ & -.08 & -.01 & -.09 & .09 \\
\hline GCI & -.06 & -.11 & -.04 & .03 \\
\hline CCI & -.04 &. $.18 * *$ & -.12 &. $\mathbf{1 8}$ \\
\hline
\end{tabular}

Notes. Ina = Inattention; Hyp / Imp = Hyperactivity / Impulsivity; VC = Verbal Comprehension; PR = Perceptual Reasoning; $\mathrm{WM}=$ Working Memory; PS = Processing Speed; GCI = General Capacity Index; CCI = Cognitive Competence Index; FSIQ = Full Scale IQ

${ }^{*} p<.05 ;{ }^{* *} p<.01 ;{ }^{* * *} p<.001$

Regression analyses indicate that only the hyperactivity/impulsivity helped to explain the performance in Processing Speed for both parents and teachers (Table 3). There was a positive relationship between Processing Speed and hyperactivity/impulsivity scores, for both parents $(b=.27$; $p<.01)$ and teachers $(b=.33 ; p<.01)$. That is, the greater the number of symptoms of hyperactivity/impulsivity, the higher the performance in Processing Speed.

Analyses were repeated using medication and gender as control variables. Results were not significantly altered, although we observed a ratio increase in the same direction as expressed in Table 3. For example, once the genre was controlled, the score in Processing Speed still predicted a greater number of symptoms both for parents $(b=.30 ; p<.01)$ and teachers $(b=.37 ; p<.01)$. Regarding medication, the obtained scores were the same as in Table 3.

\subsection{Differences between Nominal TDAH Subtypes and IQ Indexes}

Differences between average scores in each of the WISC-IV indexes were explored according to the clinical subtypes of ADHD. No significant differences between subtypes were found except for Processing Speed $\left(F_{1,214}=\right.$ 14.66; $p<.001 ; d=0.52)$. For this index, the ADHD Inattentive-Type group ( $M=90.7 ; S D=12.1)$ obtained a lower average score than the ADHD Combined-Type group $(M=97.7 ; S D=14.7)$. However, there was no significant difference for CCI between groups $(F=0.96 ; p=.09)$.

\subsection{Distance between Working Memory and Processing Speed Scores as an Indicator}

For each subject, we calculated the distance between the score in Working Memory and the score in Processing Speed, and subsequently we explored the relationship of this new variable with ADHD symptoms as well as with nominal subtypes. Hereinafter, we will label this variable as "WM-PS". A positive value on this variable indicates a higher score in Working Memory than in Processing Speed, whereas a negative value indicates the contrary.

Table 3. Unique Effects of WISC-IV index on ADHD symptoms $(\mathrm{N}=216)$.

\begin{tabular}{|c|c|c|c|c|}
\hline & \multicolumn{3}{|c|}{ Predictors } \\
\hline & \multicolumn{2}{|c|}{ Family } & Ina. & Hyp/Imp \\
\hline & Ina. & Hyp/Imp & $6(S E)$ & $6(S E)$ \\
\hline VC & $\delta(S E)$ & $6(S E)$ & $-.00(.66)$ & $-.08(.29)$ \\
\hline PR & $-.08(.59)$ & $.07(.31)$ & $-.04(.64)$ & $-.06(.28)$ \\
\hline WM & $-.02(.59)$ & $.03(.31)$ & $-.02(.69)$ & $-.05(.30)$ \\
\hline PS & $-.10(.62)$ & $.02(.32)$ & $-.19(.64)^{* *}$ & $.33(.28)^{* * *}$ \\
\hline FSIQ & $-.21(.56)^{* * *}$ & $.27(.29)^{* * *}$ & $-.09(.63)$ & $.02(.28)$ \\
\hline GCI & $-.13(.56)$ & $.13(.30)$ & $-.04(.63)$ & $-.10(.28)$ \\
\hline CCI & $-.06(.58)$ & $.05(.31)$ & $-.09(.67)$ & $.21(30)^{* *}$ \\
\hline
\end{tabular}

Notes. Ina = Inattention; Hyp / Imp = Hyperactivity / Impulsivity; VC = Verbal Comprehension; PR = Perceptual Reasoning; WM = Working Memory; PS $=$ Processing Speed; GCI = General Capacity Index; CCI = Cognitive Competence Index; FSIQ = Full Scale IQ. ${ }^{*} p<.05 ;{ }^{* *} p<.01 ;{ }^{* * *} p<.001$ 
The new variable showed a normal data distribution (K-S $p>.05)$. Correlation with age was significant but low $(r=.15$; $p<.05)$. No significant differences by gender or by medication were found. We held partial correlations (age as a constant), and we found that there was a negative correlation between the WM-PS variable and the number of hyperactivity/impulsivity symptoms, both for parents ( $r=$ $-.17 ; p<.01)$ and teachers $(r=-.24 ; p<.01)$. In applying a regression analysis, it was found that the hyperactivity/impulsivity symptoms predicted the value of the MT-VP variable through a negative relationship for parents $(b=-.23 ; p<.001)$ and for teachers $(b=-.31 ; p<.001)$. That is, the greater the number of hyperactivity/impulsivity symptoms, the lower the distance between Working Memory Index and Processing Speed Index.

The mean differences in the WM-PS variable regarding the ADHD nominal groups were also significant $\left(F_{1,214}=\right.$ 17.84; $p<.001 ; d=0.59$ ), with the ADHD Inattentive-Type group $(M=0.55 ; S D=15.6)$ showing a lower score than the ADHD Combined-Type score $(M=-9.22 ; S D=17.4)$.

\section{Discussion}

Starting from previous studies which suggest the existence of a cognitive profile obtained by WISC-IV, which is characteristic of subjects with ADHD [21, 26] the aim of this research was to explore to what extent the disorder's main dimensions, inattention and hyperactivity/impulsivity, relate to the principal indexes of the WISC-IV psychometric test. According to the hypothesis proposed in the study, a relationship between ADHD dimensions and the cognitive pattern obtained in the WISC-IV indexes was found, although this relationship was limited to the Processing Speed Index and, to a lesser extent, to the Cognitive Proficiency Index. In light of the results, we propose a new variable that is defined by the difference between Working Memory Index and Processing Speed Index. This variable shows a negative relationship that is related to hyperactivity/impulsivity, so that the greater the symptomatology of this dimension, the lower the variable value.

In line with previous research, no differences were found between the nominal groups and the General Ability Index (GAI), Verbal Comprehension Index (VCI) and Perceptual Reasoning Index (PRI) [17, 20, 24, 25].

In addition, the obtained results are consistent with other studies, linking Processing Speed Index (PSI) and ADHD predominantly Inattentive-Subtype [24, 28]. Thaler [21] found that the cluster characterized by mean scores in Verbal Comprehension, Perceptual Reasoning and Working Memory, and a low score in Processing Speed Index, showed a high level of attention deficit symptoms.

Similarly, our results are also consistent with those obtained by [29], who concluded that Processing Speed was impaired in subjects with ADHD Inattentive-Type but not in those diagnosed with ADHD Combined-Type. On the other hand, a large number of studies suggest that, in ADHD, Working Memory would be more affected than Processing Speed [30-31]. Since our study lacks a control sample, we cannot assess whether the Working Memory Index for the ADHD-diagnosed group would be significantly lower than the Working Memory Index for the control group. However, our study explores the relationships between ADHD dimensions and the indexes of WISC-IV in a clinical sample. For this reason, we cannot conclude that the absence of a relationship between ADHD symptoms and Working Memory that emerges from our results could in any way contradict the results of previous studies. What we can certainly affirm is that attention deficit disorder affects negatively the Processing Speed while the hyperactivity/impulsivity symptoms would have the opposite effect. This relationship could explain the results of studies which suggest that Processing Speed is less affected than Working Memory in ADHD, as these studies include different profiles of the disorder in the clinical sample. In this sense, the hyperactivity/impulsivity symptoms in ADHD clinical profiles that include this dimension would have a "corrector" impact on the Processing Speed Index, causing inconsistent results among the different studies within the body of literature that deal with this.

Future research should explore to what extent the presence of hyperactivity/impulsivity symptoms in clinical samples modulates the performance in Working Memory and Processing Speed indexes, and whether a lower score in the latter characterizes the ADHD predominantly Inattentive-Type profile. In the same way, future research should provide empirical evidence with respect to the hypothesis suggesting that the cognitive profile of ADHD would not be defined by a low score in Working Memory or Processing Speed with respect to the normative sample, but by the relationship between those indexes within the specific profile of the evaluated subject.

\section{Conclusions}

The results of this study provide empirical evidence for the hypothesis of the existence of a relationship between ADHD dimensions (inattention and hyperactivity/impulsivity) and cognitive profiles obtained through the application of WISC-IV. From a dimensional approach, a greater attention deficit predicted a worse performance in Processing Speed Index, while higher scores in hyperactivity/impulsivity had the reverse effect. No predictive relationship between ADHD symptoms in any of the other WISC-IV major indexes (Verbal Comprehension Index and Perceptual Reasoning Index) was found. In contrast, for processing speed, the result was lower in the ADHD predominantly Inattentive-Type group than in the ADHD Combined-Type group, taking into account that the score in both indexes was lower than the score obtained for the same subject in the 
other indices that were not affected by ADHD. Based on these data, the present authors propose a new variable that is defined by the difference between the Working Memory Index and Processing Speed Index which would open up research in the study of ADHD cognitive profiles, because it focuses on the relationship between the WISC indexes rather than on comparisons with the normative group.

\section{REFERENCES}

[1] DuPaul, G., Weyandt, L., O'Dell, S., \& Varejao, M. (2009). College students with ADHD: Current status and future directions. Journal of Attention Disorders, 13, 234-250.http://dx.doi.org/10.1177/1087054709340650.

[2] American Psychiatric Association. (2013). Diagnostic and Statistical Manual of Mental Disorders (DSM-5. 5th Edn). Washington, DC: American Psychiatric.

[3] Barkley, R., Fischer, M., Smallish, L., \& Fletcher, K. (2006). Young adult outcome of hyperactive children: Adaptive functioning in major life activities. Journal of the American Academy of Child and Adolescent Psychiatry (45), 192-202.

[4] Berger, I. (2011). Diagnosis of attention deficit hyperactivity disorder: much ado about something. Isr. Med. Assoc., 13, 571-574.

[5] Schonwald, A., \& Lechner, E. (2006). Attention deficit/hyperactivity disorder: complexities and controversies. Current Opinion in Pediatrics, 18, 189-195. http://dx.doi.org/10.1097/01.mop.0000193302.70882.70.

[6] Wolraich, M., Brown, L., Brown, R., DuPaul, G., Earls, M., \& Feldman, H. (2011). Subcommittee on attention-deficit/hyperactivity disorder; steering committee on quality improvement and management, adolescents. Pediatrics, 128, 1007-1022. http://dx.doi.org/10.1542/peds.2011-2654.

[7] Holmberg, K., \& Bölte, S. (2014). Do symptoms of ADHD at ages 7 and 10 predict academic outcome at age 16 in the general population? Journal of Attention Disorders, 18(8), 635-645.http://dx.doi.org/10.1177/1087054712452136.

[8] Miranda, A., Berenguer, C., Colomer, C., \& Roselló, R. (2014). Influence of the symptoms of Attention Deficit Hyperactivity Disorders (ADHD) and comorbid disorders on functioning in adulthood. Psicothema, 26(4), 471-476.

http://dx.doi.org/10.7334/psicothema2014.121.

[9] Skounti, G., Sikström, S., \& Smart, A. (2007). Listen to the noise is beneficial for cognitive performance in ADHD. Journal of Child Psychology and Psychiatry, 48, 840-847. http://dx.doi.org/10.1111/j.1469-7610.2007.01749.x.

[10] Capodieci, A., Gola, M. L., Cornoldi, C., \& Re, A. M. (2017). Effects of a working memory training program in preschoolers with symptoms of attention-deficit/hyperactivity disorder. Journal of Clinical and Experimental Neuropsychology, 1, 1-13. http://dx.doi.org/10.1080/13803395.2017.1307946.

[11] Gremillion, M. \& Martel, M. (2012). Semantic language as a mechanism explaining the association between ADHD symptoms and reading a mathematics underachievement. Journal of Abnormal Child Psychology, 40(8), 1339-1349. http://dx.doi.org/10.1007/s10802-012-9650-7.

[12] Jacobson, L., Ryan, M., Martin, R., Ewen, J., Mostofsky, S., Denckla, M., \& Mahone, M. (2011). Working memory influences processing speed and reading fluency in ADHD. Child Neuropsychology: A Journal on Normal and Abnormal Development in Childhood and Adolescence, 17(3), 209-224. http://dx.doi.org/10.1080/09297049.2010.532204.

[13] Kasper, L., Alderson, R., \& Hudec, K. (2012). Moderators of working memory deficits in children with attention-deficit/hyperactivity disorder (ADHD): A meta-analytic review. Clinical Psychology Review, 32, 605-617. http://dx.doi.org/doi: 10.1016/j.cpr.2012.07.001.

[14] Mattfeld, A. T., Whitfield-Gabrieli, S., Biederman, J., Spencer, T., Brown, A., Fried, R., \& Gabrieli, J. D. E. (2016). Dissociation of working memory impairments and attention-deficit/hyperactivity disorder in the brain. NeuroImage : Clinical, 10, 274-282. http://doi.org/10.1016/j.nicl.2015.12.003.

[15] Huang, F., Sun, L., Qian, Y., Liu, L., Ma, Q.-G., Yang, L., Cheng, J., Cao, Q., Su, Y., Gao, Q., Wu, Z., Li, H., Qian, Q., \& Wang, Y.-F. (2016). Cognitive Function of Children and Adolescents with Attention Deficit Hyperactivity Disorder and Learning Difficulties: A Developmental Perspective. Chinese Medical Journal, 129(16), 1922-1928. http://doi.org/10.4103/0366-6999.187861.

[16] Schreiber, J. E., Possin, K. L., Girard, J. M., \& Rey-Casserly, C. (2014). Executive Function in Children with Attention Deficit/Hyperactivity Disorder: the NIH EXAMINER battery. Journal of the International Neuropsychological Society: JINS, 20(1), 41-51.http://doi.org/10.1017/S13556177130011 00 .

[17] Shimoni, M., Engel, B., \& Tirosh, E. (2012). Executive dysfunctions among boys with Attention Deficit Hyperactivity Disorder (ADHD): Performance-based test and parents report. Research in Developmental Disabilities, 33, 858-865. http://dx.doi.org/10.1016/j.ridd.2011.12.014.

[18] Mayes, S. \& Calhoun, S. (2006). WISC-IV and WISC-III profiles in children with ADHD. Journal of Attention Disorders, 9, 486-493.http://dx.doi.org/10.1177/1087054705283616.

[19] Thaler, N., Allen, D., McMurray, J., \& Mayfield, J. (2010). Sensitivity of the test of memory and learning to attention and memory deficits in children with ADHD. Clinical Neuropsychologist, 24(2), 246-264. http://dx.doi.org/10.1080/13854040903277305.

[20] Karatekin, C., Markiewicz, S., \& Siegel, M. (2003). A preliminary study of motor problems in children with attention-deficit/hyperactivity disorders. Perceptual and Motor Skills, 97(3), 1267-1280. http://dx.doi.org/10.2466/pms.2003.97.3f.1267.

[21] Thaler, N., Bello, D., \& Etcoff, L. (2013). WISC-IV profiles are associated with differences in symptomatology and outcome in children with ADHD. Journal of Attention Disorders, 17(4), 291-301. http://dx.doi.org/10.1177/1087054711428806.

[22] Gómez, R., Vance, A., \& Watson, S. (2016). Structure of the Wechsler Intelligence Scale for Children-Fourth Edition in a 
group of children with ADHD. Frontiers in Psychology, 7, 737. https://doi.org/10.3389/fpsyg.2016.00737.

[23] Styck, K., \& Watkins, M. (2014). Structural validity of the WISC-IV for students with ADHD. Journal of Attention Disorders, 1-8. http://dx.doi.org/10.1177/1087054714553052.

[24] Yang, P., Cheng, C., Chang, C., Liu, T., Hsu, H., \& Yen, C. (2013). Wechsler Intelligence Scale for Children 4th edition-Chinese version index scores in Taiwanese children with attention-deficit/hyperactivity disorder. Psychiatry and Clinical Neurosciences, 67, 83-91. http://dx.doi.org/ 10.1111pcn.12014.

[25] Fenollar, J., Navarro, I., González, C., \& García, J. (2015). Cognitive profile for children with ADHD by using WISC-IV: subtype differences? Revista Psicodidáctica, 20(1), 157-176. http://dx.doi.org/10.1387/RevPsicodidact.12531.

[26] Devena, S., \& Watkins, M. (2012). Diagnostic utility of WISC-IV general abilities and cognitive proficiency index difference scores among children with ADHD. Journal of Applied School Psychology, 28,

133-154.http://dx.doi.org/10.1080/15377903.2012.669743.

[27] Flanagan, D., \& Kaufman, A. (2009). Claves para la evalaución con WISC-IV [Essentials of WISC-IV assessment].México: Manual Moderno.

[28] Solanto, M. V., Gilbert, S. N., Raj, A., Zhu, J., Pope-Boyd, S., \& Stepak, B. (2007). Neurocognitive functioning in ADHD, predominantly inattentive and combined subtypes. Journal of Abnormal Child Psychology, 35, 729-744.

[29] Goodman, R. (1997). The Strengths and Difficulties Questionnaire: A Research Note. Journal of Child Psychology and Psychiatry, 38, 581-586.
[30] Goodman, R. (2001). Psychometric properties of the strengths and difficulties questionnaire. Journal of the American Academy of Child and Adolescent Psychiatry, 4(11), 1337-1345.

http://dx.doi.org/10.1097/00004583-200111000-00015.

[31] Ullebø, A., Posserud, M., Heiervang, E., Gillberg, C., \& Obel, C. (2011). Screening for the attention deficit hyperactivity disorder phenotype using the strength and difficulties questionnaire. European Child \& Adolescent Psychiatry, 20, 451-458. http://dx.doi.org/1010.1007/s00787-011-0198-9.

[32] DuPaul, G., Power, T., Anastopoulos, A. \& Reid, R. (1998). ADHD Rating Scale-IV: checklists, norms, and clinical interpretation. New York: Guilford.

[33] Conners, C. K. (2008). Conners 3rd edition manual. Toronto, Ontario, Canada: Multi-Health Systems.

[34] Mayes, S., Calhoun, S., Chase, G., Mink, D., \& Stagg, R. (2009). ADHD subtypes and co-occurring anxiety, depression, and oppositional-defiant disorder: differences in Gordon diagnostic system and Wechsler's working memory and processing speed index scores. Journal of Attention Disorders, 12(6), 540-550. http://dx.doi.org/10.1177/1087054708320402.

[35] González, P., Rodríguez, C., Cueli, M., Cabeza, L., \& Álvarez, L. (2014). Math Competence and Executive Control Skills in Students with Attention Deficit/Hyperactivity Disorder and Mathematics Learning Disabilities. Revista de Psicodidáctica, 19(1), 125-143. http://dx.doi.org/doi:10.1387/RevPsicodidac.7510.

[36] Passolunghi, M., \& Cornoldi, C. (2008). Working memory failures in children with arithmetical difficulties. Children Neuropsychology, 14(5), 387-400. http://dx.doi.org/10.1080/09297040701566662. 